\title{
Resonances of the helium atom in a strong magnetic field
}

\author{
Armin Lühr, ${ }^{1, *}$ Omar-Alexander Al-Hujaj, ${ }^{1}$ and Peter Schmelcher ${ }^{1,2, \dagger}$ \\ ${ }^{1}$ Theoretische Chemie, Institut für Physikalische Chemie, Universität Heidelberg, INF 229, 69120 Heidelberg, Germany \\ ${ }^{2}$ Physikalisches Institut, Universität Heidelberg, Philosophenweg 12, 69120 Heidelberg, Germany
}

(Received 9 October 2006; published 3 January 2007)

\begin{abstract}
We present an investigation of the resonances of a doubly excited helium atom in a strong magnetic field covering the regime $B=0-100$ a.u. A full-interaction approach which is based on an anisotropic Gaussian basis set of one-particle functions being nonlinearly optimized for each field strength is employed. Accurate results for a total of 17 resonances below the threshold consisting of $\mathrm{He}^{+}$in the $N=2$ state are reported in this work. This includes states with total magnetic quantum numbers $M=0,-1,-2$ and even $z$ parity. The corresponding binding energies are compared to approximate energies of two-particle configurations consisting of two hydrogenlike electrons in the strong-field regime, thereby providing an understanding of the behavior of the energies of the resonances with varying field strength.
\end{abstract}

DOI: 10.1103/PhysRevA.75.013403

PACS number(s): 32.60.+i, 32.30.-r, 31.15.Ar

\section{INTRODUCTION}

During the last years the number of observed magnetic white dwarfs (MWD's) with magnetic field strengths $B$ between a few and $10^{5} \mathrm{~T}$ has grown rapidly. Including the ESO [44,1] and SDSS [45,2,3] surveys there are about 170 MWD's detected [4] and cataloged [46]. Even stronger fields have been found in the vicinity of neutron stars $\left(B \approx 10^{7}-10^{9} \mathrm{~T}\right)[5]$. Therefore, the behavior and properties of matter exposed to strong magnetic fields are of immediate interest. In this context, atomic physics plays a major role with respect to analyzing the atmospheres of these astronomical objects. Spectra resulting from theoretical investigations are a key ingredient in order to identify atomic (or molecular) species in the atmospheres of MWD's. Beyond this they allow one to gain information on the magnetic field strength and its landscape of the object under investigation.

Most of the literature on atoms in strong magnetic fields deals with the hydrogen atom which has also become a paradigm for a low-dimensional chaotic system [6]. Already in the 1930s it was studied employing perturbation theory in the low-field regime $\left(B \lesssim 2 \times 10^{3} \mathrm{~T}\right)$ [7] and later on also in the high-field regime. But it was not until 1984 that exact energies of the hydrogen atom for the complete magnetic field range $0 \leqslant B \leqslant 5 \times 10^{8} \mathrm{~T}$ were available [8-10]. The calculation of accurate extensive data was made possible by the advent of supercomputers. It was the accuracy and completeness of this new quantum mechanical data which permitted, after 40 years of speculation, an understanding of the mysterious absorption features in the spectrum of the MWD Grw $+70^{\circ} 8247$. They could be explained in 1985 as a hydrogen spectrum in a magnetic field with a polar value of 3.2 $\times 10^{4} \mathrm{~T}[11-13]$. In the following decades a great number of MWD's with the same type of spectrum have been identified [13].

\footnotetext{
*Present address: Institut für Physik, Humboldt-Universität zu Berlin, Hausvogteiplatz 5-7, D-10117 Berlin, Germany. Electronic address: Armin.Luehr@physik.hu-berlin.de

${ }^{\dagger}$ Electronic address: Peter.Schmelcher@pci.uni-heidelberg.de
}

In spite of this great success, there were also spectra of MWD's which could not be explained in terms of hydrogen lines. Therefore, also results of atoms with more than one electron are needed. Ten years ago knowledge about atoms with more than one electron in strong magnetic fields was still very sparse. Even in the case of two-electron atoms the investigations covered only much smaller parts of the spectrum compared to the case of the hydrogen atom and their accuracy was considerably poorer. At the end of the 1990s, sufficiently accurate and extensive data for the helium atom in the magnetic field regime $0 \leqslant B \leqslant 2.35 \times 10^{7} \mathrm{~T}[14-17]$ and later also for $2.35 \times 10^{7} \mathrm{~T} \leqslant B \leqslant 2.35 \times 10^{9} \mathrm{~T} \quad[18]$ emerged. These data consist exclusively of bound-state properties and the corresponding transitions.

Employing these data, it is possible to identify astronomical spectra of strongly magnetized helium atmospheres. In particular the interpretation of the absorption features in the spectrum of the MWD GD 229, one of the major remaining mysteries for more than 25 years, could be solved. It was the first high-field MWD with an identified helium atmosphere [19].

Nevertheless, the spectra of several MWD's with an estimated hydrogen or helium atmosphere show major absorption features that remain unexplained. There were indications to explain the unresolved features with bound-free and freefree transitions but also with spectral components of heavier elements or of ions. However, the literature concerning these topics is comparably sparse. In order to shed light on this issue there were efforts to calculate bound-free opacities for the hydrogen atom [20]. More recently, extensive and accurate calculations on the bound-state spectrum of heavier elements like lithium [21] and beryllium [22] in strong magnetic fields have been accomplished. However, these newer results have not been able to explain the unresolved absorption features in question.

In view of the above it is of particular interest to explore the doubly excited states-i.e., the resonances of helium in strong magnetic fields-which have not been considered to date in the literature. It is the aim of this work to provide a step towards the understanding of doubly excited helium atoms in strong magnetic fields and to provide the first data for the corresponding resonances. 
TABLE I. Correspondence table between the field (2) and field-free (4) notation for the doubly excited helium atom for $B=0$. The field-free energy values are taken from the literature.

\begin{tabular}{|c|c|c|c|c|c|}
\hline $\begin{array}{l}S=0: \\
\text { Energy }\end{array}$ & $\begin{array}{c}\text { Field-free } \\
\left|L^{\Pi}{ }_{N}(K, T)_{n}^{A}\right|\end{array}$ & $\begin{array}{l}\text { Field } \\
{ }_{N} \nu M^{\Pi_{z}}\end{array}$ & $\begin{array}{c}S=1: \\
\text { Energy }\end{array}$ & $\begin{array}{c}\text { Field-free } \\
\left|L^{\Pi}{ }_{N}(K, T)_{n}^{A}\right|\end{array}$ & $\begin{array}{l}\text { Field } \\
{ }_{N} \nu M^{\Pi_{z}}\end{array}$ \\
\hline $0.77786764[35]$ & ${ }^{1} S^{e}{ }_{2}(1,0)_{2}^{+}$ & ${ }_{2} 1^{1} 0^{+}$ & $0.76049239[36]$ & ${ }^{3} P_{2}^{o}(1,0)_{2}^{+}$ & ${ }_{2} 1{ }^{3} 0^{-}$ \\
\hline $0.7019457[37]$ & ${ }^{1} D^{e}{ }_{2}(1,0)_{2}^{+}$ & $\begin{array}{l}2^{2}{ }^{1} 0^{+} \\
{ }_{2} 1^{1}( \pm 1)^{-} \\
{ }_{2} 1^{1}( \pm 2)^{+}\end{array}$ & $0.7105002[41]$ & ${ }^{3} P^{e}{ }_{2}(0,1)_{2}^{+}$ & $\begin{array}{l}{ }_{2} 1^{3}( \pm 1)^{+} \\
{ }_{2} 1^{3} 0^{+} \\
{ }_{2} 1^{3}( \pm 1)^{-}\end{array}$ \\
\hline $0.69313495[36]$ & ${ }^{1} P_{2}^{o}(0,1)_{2}^{+}$ & $\begin{array}{l}{ }_{2} 1^{1} 0^{-} \\
{ }_{2} 1^{1}( \pm 1)^{+}\end{array}$ & $\begin{array}{l}0.6025775[35] \\
0.5846723[36]\end{array}$ & $\begin{array}{l}{ }^{3} S_{2}^{e}(1,0)_{3}^{-} \\
{ }^{3} P_{2}^{o}{ }_{2}(1,0)_{3}^{+}\end{array}$ & $\begin{array}{l}2^{2}{ }^{3} 0^{+} \\
2^{2}{ }^{3} 0^{-}\end{array}$ \\
\hline $0.62192725[35]$ & ${ }^{1} S^{e}{ }_{2}(-1.0)_{2}^{+}$ & ${ }_{2} 3{ }^{1} 0^{+}$ & & & ${ }_{2} 2^{3}( \pm 1)^{+}$ \\
\hline $0.5970738[36]$ & ${ }^{1} P_{2}^{o}(1,0)_{3}^{-}$ & $\begin{array}{l}2^{2}{ }^{1} 0^{-} \\
2^{2}{ }^{1}( \pm 1)^{+}\end{array}$ & $0.58378427[37]$ & ${ }^{3} D^{e}{ }_{2}(1,0)_{3}^{-}$ & $\begin{array}{l}2^{3}{ }^{3} 0^{+} \\
2^{2}{ }^{3}( \pm 1)^{-}\end{array}$ \\
\hline $0.58989468[35]$ & ${ }^{1} S^{e}{ }_{2}(1,0)_{3}^{+}$ & $2^{4}{ }^{1} 0^{+}$ & & & ${ }_{2} 1^{3}( \pm 2)^{+}$ \\
\hline $0.58025[41]$ & ${ }^{1} P_{2}^{e}(0,1)_{3}^{-}$ & $\begin{array}{l}2^{5}{ }^{1} 0^{+} \\
{ }_{2} 1^{1}( \pm 1)^{-}\end{array}$ & $0.57903099[36]$ & ${ }^{3} P_{2}^{o}(0,1)_{3}^{-}$ & $\begin{array}{l}2^{3}{ }^{3} 0^{-} \\
{ }_{2} 3^{3}( \pm 1)^{+}\end{array}$ \\
\hline $0.569221[37]$ & ${ }^{1} D^{e}{ }_{2}(1,0)_{3}^{+}$ & $\begin{array}{l}2^{6}{ }^{1} 0^{+} \\
{ }_{2} 3^{1}( \pm 1)^{-}\end{array}$ & $0.56781[41]$ & ${ }^{3} P_{2}^{e}(0,1)_{3}^{+}$ & $\begin{array}{l}2^{4}{ }^{3} 0^{+} \\
2^{3} 3^{3}( \pm 1)^{-}\end{array}$ \\
\hline $0.564085[36]$ & ${ }^{1} P_{2}^{o}(0,1)_{3}^{+}$ & $\begin{array}{l}2^{2}{ }^{1}( \pm 2)^{+} \\
2^{3}{ }^{1} 0^{-} \\
{ }_{2} 3^{1}( \pm 1)^{+}\end{array}$ & $0.5662[41]$ & ${ }^{3} F_{2}^{o}(1,0)_{3}^{0}$ & $\begin{array}{l}2^{4}{ }^{3} 0^{-} \\
2^{4}{ }^{3}( \pm 1)^{+} \\
{ }_{2} 1^{3}( \pm 2)^{-}\end{array}$ \\
\hline $0.5638[41]$ & ${ }^{1} D_{2}^{o}(1,1)_{2}^{+}$ & $\begin{array}{l}2^{4}{ }^{1} 0^{-} \\
2^{4}{ }^{1}( \pm 1)^{+} \\
{ }_{2} 1^{1}( \pm 2)^{-}\end{array}$ & $0.560687[37]$ & ${ }^{3} D^{e}{ }_{2}(0,1)_{3}^{0}$ & $\begin{array}{l}{ }_{2} 1^{3}( \pm 3)^{+} \\
{ }_{2} 5^{3} 0^{+} \\
2^{4}{ }^{3}( \pm 1)^{-} \\
2^{2} 2^{3}( \pm 2)^{+}\end{array}$ \\
\hline
\end{tabular}

The paper is organized as follows: In Sec. II we introduce the Hamiltonian and discuss its symmetries. In Sec. III we report on our computational approach and the employed optimized basis set. Section IV begins with a comparison of calculated resonance energies with the literature in the wellknown field-free case. Subsequently the resonance energies and their behavior for different magnetic field strengths are presented and discussed. The dominant configurations contributing to the resonances are identified, allowing us to interprete them. Additionally, the widths of selected resonances are studied with increasing magnetic field strength.

\section{HAMILTONIAN AND SYMMETRIES}

Our investigation is carried out under the assumption of an infinite nuclear mass. However, by exploiting a pseudoseparation of the center-of-mass motion in a magnetic field [23-25] a scaling law can be derived which allows one to translate the data for fixed nucleus into results with a finite nuclear mass under the assumption of a vanishing pseudomomentum (see [14] and references therein). The finitenuclear-mass effects have certainly to be taken into account in the high-field regime [18]. However, in this work we focus on the intermediate-field regime and can therefore safely neglect the corresponding considerations.

Assuming the magnetic field $\boldsymbol{B}$ to point in the positive $z$ direction and for the field strength $B=|\boldsymbol{B}|$, our Hamiltonian reads (atomic units are used throughout the paper)

$$
\begin{aligned}
H= & \sum_{i=1}^{2}\left(\frac{1}{2} \boldsymbol{p}_{i}^{2}-\frac{2}{\left|\boldsymbol{r}_{i}\right|}+\frac{1}{2} B\left(l_{i}\right)_{z}+\frac{B^{2}}{8}\left(x_{i}^{2}+y_{i}^{2}\right)+B\left(s_{i}\right)_{z}\right) \\
& +\frac{1}{\left|\boldsymbol{r}_{2}-\boldsymbol{r}_{1}\right|} .
\end{aligned}
$$

The one-particle operators in Eq. (1) are the field-free kinetic energies $\frac{p_{i}^{2}}{2}$, the Coulomb potential energies $-\frac{2}{\left|r_{i}\right|}$ of the electrons in the field of the nucleus, the Zeeman terms $\frac{1}{2} B\left(l_{i}\right)_{z}$, the diamagnetic terms $\frac{B^{2}}{8}\left(x_{i}^{2}+y_{i}^{2}\right)$, and the spin energies $B\left(s_{i}\right)_{z}$. The two-particle operator $\frac{1}{\left|\boldsymbol{r}_{2}-\boldsymbol{r}_{1}\right|}$ represents the electron-electron repulsion energy. For remarks on the influence of relativistic effects and on the electron spin $g$ factor, here taken to be equal to 2 , we refer the reader to $[10,14]$.

There exist four independent quantities commuting with the Hamiltonian (1): The total spin $S^{2}$, the $z$ component $S_{z}$ of the total spin, the $z$ component $L_{z}$ of the total orbital angular momentum, and the total spatial $z$ parity $\Pi_{z}$. Therefore, the following investigations take place in a subspace of a specified symmetry-i.e., with given eigenvalues of $S^{2}, S_{z}, L_{z}$, and $\Pi_{z}$. Furthermore, we will denote the calculated states by the magnetic field spectroscopic notation

$$
{ }_{N} \nu^{2 S+1} M^{\Pi_{z}}
$$

where $2 S+1$ is the spin multiplicity, $M$ the eigenvalue of $L_{z}$, and $\nu=1,2,3, \ldots$ denotes the degree of excitation within a 
TABLE II. The relative accuracy of the energetically lowest resonances of doubly excited helium for $B=0$ in the subspaces $\Pi_{z}=+1$ and $M=0, \pm 1$ and \pm 2 with $S=0,1, S_{z}=0$, and $N=2$ are presented. The accuracies are ordered by the degree of excitation $\nu$ of their corresponding states within a given symmetry subspace (cf. Table I). The data used for comparison are indicated by reference number.

\begin{tabular}{|c|c|c|c|}
\hline$\nu$ & $\Delta E$ & $\Delta \Gamma$ & \\
\hline \multicolumn{4}{|c|}{$M=0 S=0$} \\
\hline 1 & $7.07 \times 10^{-5}$ & $1.09 \times 10^{-2}$ & [35] \\
\hline 2 & $5.41 \times 10^{-4}$ & $5.22 \times 10^{-2}$ & [37] \\
\hline 3 & $2.10 \times 10^{-3}$ & $7.03 \times 10^{-1}$ & [35] \\
\hline 4 & $7.12 \times 10^{-5}$ & $5.53 \times 10^{-2}$ & [35] \\
\hline \multicolumn{4}{|c|}{$M=0 S=1$} \\
\hline 1 & $1.25 \times 10^{-4}$ & & [41] \\
\hline 2 & $4.81 \times 10^{-5}$ & $8.13 \times 10^{-1}$ & [35] \\
\hline 3 & $2.39 \times 10^{-5}$ & & [37] \\
\hline \multicolumn{4}{|c|}{$M= \pm 1 S=0$} \\
\hline 1 & $5.24 \times 10^{-4}$ & $3.12 \times 10^{-2}$ & [36] \\
\hline 2 & $4.19 \times 10^{-5}$ & $4.83 \times 10^{-1}$ & [36] \\
\hline 3 & $2.30 \times 10^{-4}$ & $1.30 \times 10^{-1}$ & [36] \\
\hline 4 & $7.50 \times 10^{-4}$ & & [41] \\
\hline \multicolumn{4}{|c|}{$M= \pm 1 S=1$} \\
\hline 1 & $6.84 \times 10^{-5}$ & $6.59 \times 10^{-3}$ & [36] \\
\hline 2 & $3.59 \times 10^{-5}$ & $2.88 \times 10^{-1}$ & [36] \\
\hline 3 & $5.53 \times 10^{-5}$ & $3.45 \times 10^{-0}$ & [36] \\
\hline 4 & $9.18 \times 10^{-4}$ & $2.72 \times 10^{-0}$ & [41] \\
\hline \multicolumn{4}{|c|}{$M= \pm 2 S=0$} \\
\hline 1 & $5.41 \times 10^{-4}$ & $5.22 \times 10^{-2}$ & [37] \\
\hline 2 & $1.67 \times 10^{-4}$ & $5.81 \times 10^{-2}$ & [37] \\
\hline
\end{tabular}

given symmetry subspace, whereas $N$ represents the excitation of the "inner" electron. The latter is not an exact quantum number but a helpful spectroscopic label within an independent-particle picture.

\section{COMPUTATIONAL APPROACH}

The Schrödinger equation is solved by applying a full configuration-interaction (full CI) approach. For our calculations we use an anisotropic Gaussian basis set which has been put forward by Schmelcher and Cederbaum [26] for the purpose of investigating atoms and molecules in strong magnetic fields. This one-particle basis set has been successfully applied to several atoms, ions, and molecules [14-16,18,21,22,27-30]. The basis functions are optimized for each field strength and each symmetry separately using a nonlinear optimization procedure described in [14,18]. A two-particle basis set within a subspace $\left(M, \Pi_{z}\right)$ is constructed by selecting configurations of two optimized oneparticle orbitals respecting the symmetries. The full CI approach leads to a generalized eigenvalue problem in which
TABLE III. Correspondence between the spectroscopic field and field-free notation for vanishing magnetic field. Additionally, for a strong magnetic field the corresponding approximate configurations of a $\mathrm{He}^{+}$and a $\mathrm{H}$ electron are presented. Configuration marked by $\dagger$ are intrashell states which quantitatively do not approximate the energies well.

\begin{tabular}{lcc}
\hline \hline Field not. & Field-free not. & App. config. \\
$B \geqslant 0$ & $B=0$ & $B \rightarrow \infty$ \\
\hline $1^{1} 0^{+}$ & ${ }^{1} S^{g}{ }_{2}(1,0)_{2}^{+}$ & $2 p_{0} 2 p_{0}{ }^{\dagger}$ \\
$2{ }^{1} 0^{+}$ & ${ }^{1} D^{g}{ }_{2}(1,0)_{2}^{+}$ & $2 p_{0} 3 p_{0}$ \\
$3{ }^{1} 0^{+}$ & ${ }^{1} S^{g}{ }_{2}(-1,0)_{2}^{+}$ & $2 p_{0} 4 f_{0}$ \\
$4^{1} 0^{+}$ & ${ }^{1} S^{g}{ }_{2}(1,0)_{3}^{+}$ & $2 p_{0} 4 p_{0}$ \\
\hline $1^{3} 0^{+}$ & ${ }^{3} P^{g}{ }_{2}(0,1)_{2}^{+}$ & $2 p_{0} 3 p_{0}$ \\
$2^{3} 0^{+}$ & ${ }^{1} S^{g}{ }_{2}(1,0)_{3}^{-}$ & $2 p_{0} 4 f_{0}$ \\
$3^{3} 0^{+}$ & ${ }^{1} D^{g}{ }_{2}(1,0)_{3}^{-}$ & $2 p_{0} 4 p_{0}$ \\
\hline $1^{1}(-1)^{+}$ & ${ }^{1} P^{u}{ }_{2}(0,1)_{2}^{+}$ & $2 p_{-1} 2 s_{0}$ \\
$2^{1}(-1)^{+}$ & ${ }^{1} P^{u}{ }_{2}(1,0)_{3}^{-}$ & $2 p_{-1} 3 d_{0}$ \\
$3^{1}(-1)^{+}$ & ${ }^{1} P^{u}{ }_{2}(0,1)_{3}^{+}$ & $2 p_{-1} 3 s_{0}$ \\
$4^{1}(-1)^{+}$ & ${ }^{1} D^{u}{ }_{2}(1,1)_{2}^{+}$ & $2 p_{-1} 4 d_{0}$ \\
\hline $1^{3}(-1)^{+}$ & ${ }^{3} P^{u}{ }_{2}(1,0)_{2}^{+}$ & $2 p_{-1} 3 d_{0}$ \\
$2^{3}(-1)^{+}$ & ${ }^{3} P^{u}{ }_{2}(1,0)_{3}^{+}$ & $2 p_{-1} 3 d_{0}$ \\
$3^{3}(-1)^{+}$ & ${ }^{3} P^{u}{ }_{2}(0,1)_{3}^{-}$ & $2 p_{-1} 3 s_{0}$ \\
$4^{3}(-1)^{+}$ & ${ }^{3} F^{u}{ }_{2}(1,0)_{3}^{0}$ & $2 p_{-1} 4 d_{0}$ \\
\hline $1^{1}(-2)^{+}$ & ${ }^{1} D^{g}{ }_{2}(1,0)_{2}^{+}$ & $2 p_{-1} 2 p_{-1}{ }^{\dagger}$ \\
$2^{1}(-2)^{+}$ & ${ }^{1} D^{g}{ }_{2}(1,0)_{3}^{+}$ & $2 p_{-1} 3 p_{-1}$ \\
\hline \hline & & \\
\hline
\end{tabular}

all matrix elements can be calculated analytically and evaluated efficiently $[14,15]$. Further technical aspects are discussed in $[14,18,29]$.

For the investigation in this paper we use the complexscaling or complex-rotation method. This means that all spatial coordinates $\boldsymbol{r}$ in the Hamiltonian are formally replaced with $\boldsymbol{r} e^{i \theta}$ (cf. [31,32]). This leads to a non-Hermitian Hamiltonian with complex eigenvalues $W=E-\frac{i}{2} \Gamma$ with energy $E$ and resonance width $\Gamma$ and to a complex-symmetric matrix representation. The energies of the bound states are unaffected by this transformation, while the different continua are rotated about their respective thresholds by the angle $-2 \theta$ from the real axis into the complex energy plane. The hidden resonances of the Hamiltonian in the continuum, associated with complex eigenvalues $W$, are revealed if a sufficiently large value of $\theta$ is chosen.

It is one major advantage of the complex-scaling method that the sophisticated numerical code developed for bound atom systems can be directly "translated" to the case of unbound resonances. However, the complex eigenvalues $W$ of the resonances are only independent of the rotation angle $\theta$ if the basis set is complete, which is not the case in any approximate numerical investigation. Therefore, the rotated Hamiltonian has to be calculated for different values of $\theta$. The complex variational theorem and the complex Hellmann-Feynman theorem provide a criterion for choosing an optimum resonance parameter $\theta$ within a given basis set [33]: 
TABLE IV. Calculated energies $E$ and resonance widths $\Gamma$ of resonances of the subspace ${ }^{1}(0)^{+}$and $N=2$ for different magnetic field strengths $B$ : $1^{1}(0)^{+}$and $2^{1}(0)^{+}$[field-free ${ }^{1} S^{e}{ }_{2}(1,0)_{2}^{+}$and $\left.{ }^{1} D^{e}{ }_{2}(1,0)_{2}^{+}\right]$.

\begin{tabular}{|c|c|c|c|c|}
\hline \multirow[b]{2}{*}{$B$} & \multicolumn{2}{|c|}{$1^{1}(0)^{+}$} & \multicolumn{2}{|c|}{$2^{1}(0)^{+}$} \\
\hline & $-E$ & $\Gamma / 2$ & $-E$ & $\Gamma / 2$ \\
\hline 0 & $0.77786[35]$ & 0.002271 & $0.70195[37]$ & 0.001181 \\
\hline 0 & 0.77781 & 0.002246 & 0.70157 & 0.001121 \\
\hline 0.0002 & 0.77762 & 0.002299 & 0.69948 & 0.001211 \\
\hline 0.0005 & 0.77763 & 0.002291 & 0.6995 & 0.001217 \\
\hline 0.001 & 0.77802 & 0.002296 & 0.70093 & 0.001619 \\
\hline 0.002 & 0.77781 & 0.002283 & 0.70116 & 0.001206 \\
\hline 0.005 & 0.77776 & 0.002312 & 0.70112 & 0.001193 \\
\hline 0.007 & 0.77772 & 0.002311 & 0.70064 & 0.001163 \\
\hline 0.01 & 0.77758 & 0.002249 & 0.70098 & 0.000523 \\
\hline 0.02 & 0.77691 & 0.002683 & 0.70006 & 0.00225 \\
\hline 0.04 & 0.77348 & 0.001094 & 0.68867 & 0.000155 \\
\hline 0.05 & 0.7726 & 0.002337 & 0.69688 & 0.000952 \\
\hline 0.08 & 0.76462 & 0.002319 & 0.68842 & \\
\hline 0.1 & 0.75772 & 0.002487 & 0.68534 & 0.001109 \\
\hline 0.16 & 0.72982 & 0.002751 & 0.66255 & 0.001122 \\
\hline 0.4 & 0.57765 & 0.002779 & 0.48461 & 0.002578 \\
\hline 0.5 & 0.51019 & 0.003137 & 0.37951 & 0.002904 \\
\hline 0.8 & 0.29357 & 0.003192 & 0.03998 & 0.001906 \\
\hline 1 & 0.13985 & 0.002744 & -0.12582 & 0.00086 \\
\hline 2 & -0.70432 & 0.01068 & -0.99833 & 0.002647 \\
\hline 5 & -3.47217 & 0.005766 & -3.80865 & 0.000267 \\
\hline 10 & -8.29029 & 0.010718 & -8.65359 & 0.001035 \\
\hline 20 & -18.0834 & 0.009865 & -18.4943 & 0.00912 \\
\hline 50 & -47.8771 & 0.007483 & -48.3142 & 0.002379 \\
\hline 100 & -97.7081 & & -98.1969 & \\
\hline
\end{tabular}

$$
\left.\frac{d W(\theta)}{d \theta}\right|_{\theta_{\mathrm{opt}}}=0 .
$$

To find that optimum $\theta_{\text {opt }}$, one also has to vary the imaginary part of $\theta$, which can be interpreted as a real scaling parameter of the wave functions. Obviously, the criterion (3) is only fulfilled approximately since we can only calculate finite differences between eigenvalues belonging to Hamiltonians with a different value of the rotation angle $\theta$.

In our investigation the diagonalization of the rotated Hamiltonian is carried out for about 50-80 different values of $\theta$. The used basis-set size varies from 4000 to 6000 twoparticle configurations. This results in a computational effort of approximately 30-200 h CPU time on a powerful PC for each magnetic field strength and symmetry subspace.

\section{RESULTS}

In principle, one distinguishes two kinds of excitations of the helium atom using the independent-particle picture. On
TABLE V. Calculated energies $E$ and resonance widths $\Gamma$ of resonances of the subspace ${ }^{1}(0)^{+}$and $N=2$ for different magnetic field strengths $B$ : $3{ }^{1}(0)^{+}$and $4^{1}(0)^{+}$[field-free ${ }^{1} S^{e}{ }_{2}(-1,0)_{2}^{+}$and $\left.{ }^{1} S_{2}^{e}(1,0)_{3}^{+}\right]$.

\begin{tabular}{|c|c|c|c|}
\hline \multirow[b]{2}{*}{$B$} & \multirow{2}{*}{$\begin{array}{c}3^{1}(0)^{+} \\
-E\end{array}$} & \multicolumn{2}{|c|}{$4^{1}(0)^{+}$} \\
\hline & & $-E$ & $\Gamma / 2$ \\
\hline 0 & $0.62193[35]$ & $0.58989[35]$ & 0.000681 \\
\hline 0 & 0.62062 & 0.58985 & 0.000644 \\
\hline 0.0002 & 0.61347 & 0.58965 & 0.000655 \\
\hline 0.0005 & 0.62085 & 0.58960 & 0.000609 \\
\hline 0.001 & 0.62044 & 0.58794 & 0.001025 \\
\hline 0.002 & 0.62044 & 0.58984 & 0.000670 \\
\hline 0.005 & 0.62085 & 0.58960 & 0.000710 \\
\hline 0.007 & 0.62048 & 0.58941 & 0.000699 \\
\hline 0.01 & 0.62009 & 0.58849 & 0.000924 \\
\hline 0.02 & 0.61873 & 0.58637 & 0.000377 \\
\hline 0.04 & 0.61373 & 0.57662 & 0.000755 \\
\hline 0.05 & 0.61368 & 0.57308 & 0.000852 \\
\hline 0.08 & 0.60268 & 0.55647 & 0.000914 \\
\hline 0.1 & 0.59495 & 0.54606 & 0.000625 \\
\hline 0.16 & 0.56150 & 0.50973 & 0.000372 \\
\hline 0.4 & 0.36064 & 0.34611 & 0.000468 \\
\hline 0.5 & 0.27335 & 0.25830 & 0.000402 \\
\hline 0.8 & 0.03153 & -0.01283 & 0.000086 \\
\hline 1 & -0.17336 & -0.19726 & 0.001278 \\
\hline 2 & -1.05301 & -1.06606 & 0.000748 \\
\hline 5 & -3.86318 & -3.88367 & \\
\hline 10 & -8.71004 & -8.73188 & \\
\hline 20 & -18.55700 & -18.58471 & 0.000631 \\
\hline 50 & -48.37604 & -48.41477 & 0.000049 \\
\hline 100 & -98.19686 & -98.29527 & \\
\hline
\end{tabular}

the one hand, there are the single excitations of the helium atom. Their "inner" electron occupies predominantly the one-particle ground state of $\mathrm{He}^{+}$. The second "outer" electron carries, like a hydrogenic electron, the angular momentum alone. All bound states are of this kind. On the other hand, there are the doubly excited helium states where the inner electron is excited, too. They appear as resonances in the continuum of the singly excited helium states. The degree of the inner and outer excitation is denoted by $N$ with $N=1,2, \ldots$ and by $n$ with $n \geqslant N$, respectively. States with the same inner excitation $N$ form Rydberg series converging energetically to the threshold $I_{N}$. These thresholds correspond to the energies of an excited helium ion $\mathrm{He}^{+}$in the $N$ th energy level.

\section{A. Resonances in the field-free case}

Doubly excited (DE) helium has been an active research topic for the last 40 years, from both numerical and analytical standpoints (cf. review [34]). Major contributions to the calculation of the energy levels were provided by, e.g., 
TABLE VI. Calculated energies $E$ of resonances of the subspace ${ }^{3}(0)^{+}$and $N=2$ for different magnetic field strengths $B: 1^{3}(0)^{+}$, $2^{3}(0)^{+}$, and $3^{3}(0)^{+}$[field-free ${ }^{3} P^{e}{ }_{2}(-1,0)_{2}^{+},{ }^{3} S^{e}{ }_{2}(-1,0)_{3}^{-}$, and $\left.{ }^{3} D^{e}{ }_{2}(1,0)_{3}^{-}\right]$.

\begin{tabular}{|c|c|c|c|}
\hline$B$ & $\begin{array}{c}1^{3}(0)^{+} \\
-E\end{array}$ & $\begin{array}{c}2^{3}(0)^{+} \\
-E\end{array}$ & $\begin{array}{c}3^{3}(0)^{+} \\
-E\end{array}$ \\
\hline 0 & $0.71050[41]$ & $0.60258[35]$ & $0.58378[37]$ \\
\hline 0 & 0.71041 & 0.60255 & 0.58359 \\
\hline 0.0002 & 0.70621 & 0.60250 & 0.58331 \\
\hline 0.0005 & 0.71039 & 0.60237 & 0.58344 \\
\hline 0.001 & 0.71045 & 0.60064 & 0.58344 \\
\hline 0.002 & 0.71036 & 0.60254 & 0.58345 \\
\hline 0.005 & 0.71039 & 0.60237 & 0.58344 \\
\hline 0.007 & 0.71033 & 0.60222 & 0.58298 \\
\hline 0.01 & 0.71014 & 0.60157 & 0.58176 \\
\hline 0.02 & 0.70952 & 0.59899 & 0.58001 \\
\hline 0.04 & 0.70672 & 0.59146 & 0.57100 \\
\hline 0.05 & 0.70465 & 0.58797 & 0.56960 \\
\hline 0.08 & 0.69598 & 0.57264 & 0.54839 \\
\hline 0.1 & 0.68825 & 0.56251 & 0.53147 \\
\hline 0.16 & 0.65724 & 0.52765 & 0.48792 \\
\hline 0.4 & 0.45585 & 0.36377 & 0.30446 \\
\hline 0.5 & 0.34928 & 0.28915 & 0.22842 \\
\hline 0.8 & 0.05382 & -0.00755 & -0.01468 \\
\hline 1 & -0.11049 & -0.8276 & -0.19474 \\
\hline 2 & -0.98307 & -1.04769 & -1.07099 \\
\hline 5 & -3.78678 & -3.85651 & -3.88082 \\
\hline 10 & -8.62833 & -8.70260 & -8.72806 \\
\hline 20 & -18.47258 & -18.55026 & -18.57670 \\
\hline 50 & -48.28332 & -48.36675 & -48.40350 \\
\hline 100 & -98.16338 & -98.25919 & -98.28200 \\
\hline
\end{tabular}

Bürgers et al. ( $S$ states) [35], Ho and Bhatia ( $P$ and $D$ states) [36,37], and Bachau ( $N=3$ states) [38]. However, the data have not reached the same accuracy and completeness compared with the literature of singly excited helium. DE helium is considered as a standard model for a strongly correlated system. We use the standard notation-i.e., the correlation quantum numbers introduced by Herrick and Sinanoğlu [39] and Lin [40]:

$$
{ }^{2 S+1} L^{\Pi}(K, T)_{n}^{A} .
$$

Here $L$ is the total angular momentum and $K$ and $T$ represent angular and $A$ radial correlations.

However, the focus of this paper lies on the interaction of the atomic system in a strong magnetic field. For a finite field strength the correlation quantum numbers (4) lose their meaning. Therefore, it is instructive to consider the correspondence between the magnetic field spectroscopic notation (2) and the field-free one (4) which is done for the states studied here in Table I.

Table II provides the relative accuracy of our calculated energies $E$ and decay widths $\Gamma$ for the energetically lowest resonances in the absence of the field. The comparison was performed with the corresponding high-precision results available in the literature [35-37,41]. Generally it has to be remarked that for the states considered here, the absolute values of the energies are significantly larger than the values of the corresponding widths. The accuracy of our results is consequently much higher for the energies compared to the widths. Except for the states $3^{1} 0^{+}$and $4^{3}( \pm 1)^{+}$all calculated energies possess a relative accuracy in the range of $7.5 \times 10^{-4}-2 \times 10^{-5}$.

Obviously our approach is not best suited to describe the resonances in the absence of the external field-i.e., cannot compete with the best methods available in the literature based, e.g., on a Hylleraas basis. However, our focus is to provide first results on resonances in strong magnetic fields and in this case our anisotropic Gaussian basis set is certainly superior to existing field-free methods that cannot efficiently describe the pronounced anisotropy present in a strong field. This reflects itself also in the different symmetries for $B=0$ and $B \neq 0$.

\section{B. Resonances in a magnetic field}

\section{Dependence of the resonance energies on $M$ and $S_{z}$}

The Zeeman term of the Hamiltonian (1) depends linearly on $L_{z}$ and remains unchanged when the complex rotation is applied. Using eigenfunctions of $L_{z}$, as is done here, one arrives at

$$
\left\langle i\left|T_{\text {Zeeman }}(M)\right| j\right\rangle=\left\langle i\left|\frac{1}{2} L_{z} B\right| j\right\rangle=\frac{1}{2} M B S_{i j},
$$

with $S_{i j}$ being the overlap matrix of the two-particle states $|i\rangle$ and $|j\rangle$. The relation between two matrix elements associated with different signs of the magnetic quantum number $M$ reads as follows:

$$
\left\langle i\left|T_{\text {Zeeman }}(-M)\right| j\right\rangle=\left\langle i\left|T_{\text {Zeeman }}(M)\right| j\right\rangle-M B S_{i j} ;
$$

i.e., we obtain a shift. A similar argument holds for $S_{z}$,

$$
\left\langle i\left|T_{\text {spin }}\left(S_{z}\right)\right| j\right\rangle=\left\langle i\left|S_{z} B\right| j\right\rangle=S_{z} B S_{i j}
$$

In combination, the relation for the dependence of the complex energy $W$ on $M$ and $S_{z}$ can be put in the following way:

$$
W\left(M, S_{z}\right)=W\left(-M, S_{z}=0\right)+M B+S_{z} B .
$$

In what follows, we only present results for states belonging to subspaces with $M \leqslant 0$ and $S_{z}=0$. All energies of subspaces with $M>0$ and $S_{z} \neq 0$ are just shifted by a real energy term and can be calculated with the help of Eq. (5). Note that the resonance widths do not depend on $S_{z}$ and the sign of $M$.

\section{Resonance energies}

The results of our calculations include the energies of the energetically lowest resonance states of the subspaces with $M=0,-1,-2, \Pi_{z}=+1, S_{z}=0$, and $S=0,1$. The values of the magnetic field strength are spread over a logarithmic scale covering the range $B=0-100$ a.u. $\left(B=0-2.35 \times 10^{7} \mathrm{~T}\right)$. The resulting data are given in Tables IV-IX.

The minimal energy of a single free electron $(m \leqslant 0)$ in a magnetic field is $E_{e^{-}}(B)=B / 2$ (cf. Landau energy [42]). 
TABLE VII. Calculated energies $E$ and resonance widths $\Gamma$ of resonances of the subspace ${ }^{1}(-1)^{+}$and $N=2$ for different magnetic field strengths $B: 1^{1}(-1)^{+} 2^{1}(-1)^{+}, 3{ }^{1}(-1)^{+}$, and $4^{1}(-1)^{+}$[field-free ${ }^{1} P_{2}^{o}(0,1)_{2}^{+},{ }^{1} P_{2}^{o}(1,0)_{3}^{-},{ }^{1} P_{2}^{o}(0,1)_{3}^{+}$, and $\left.{ }^{1} D_{2}^{o}(1,1)_{2}^{+}\right]$.

\begin{tabular}{|c|c|c|c|c|c|}
\hline \multirow[b]{2}{*}{$B$} & \multicolumn{2}{|c|}{$1^{1}(-1)^{+}$} & \multirow{2}{*}{$\begin{array}{c}2^{1}(-1)^{+} \\
-E\end{array}$} & \multirow{2}{*}{$\begin{array}{c}3^{1}(-1)^{+} \\
-E\end{array}$} & \multirow{2}{*}{$\begin{array}{c}4^{1}(-1)^{+} \\
-E\end{array}$} \\
\hline & $-E$ & $\Gamma / 2$ & & & \\
\hline 0 & $0.69313[36]$ & 0.000687 & $0.59707[36]$ & $0.56408[36]$ & $0.56338[41]$ \\
\hline 0 & 0.69277 & 0.000708 & 0.59705 & 0.56395 & 0.56338 \\
\hline 0.0002 & 0.69293 & 0.000645 & 0.59716 & 0.56413 & 0.56356 \\
\hline 0.0005 & 0.69286 & 0.000541 & 0.59724 & 0.56420 & 0.56317 \\
\hline 0.001 & 0.69306 & 0.000875 & 0.59723 & 0.56518 & 0.56260 \\
\hline 0.002 & 0.69359 & 0.000530 & 0.59783 & 0.56482 & 0.56470 \\
\hline 0.005 & 0.69499 & 0.000691 & 0.59929 & 0.56611 & 0.56517 \\
\hline 0.01 & 0.69746 & 0.000599 & 0.60123 & 0.56766 & 0.56732 \\
\hline 0.02 & 0.70032 & 0.001664 & 0.60359 & 0.57098 & 0.56781 \\
\hline 0.04 & 0.70755 & 0.000589 & 0.60505 & 0.57442 & 0.56664 \\
\hline 0.08 & 0.71809 & 0.000518 & 0.59790 & 0.57637 & 0.56085 \\
\hline 0.1 & 0.71239 & 0.000749 & 0.59139 & 0.56711 & 0.54302 \\
\hline 0.16 & 0.71991 & 0.000854 & 0.58014 & 0.54826 & 0.53355 \\
\hline 0.4 & 0.64988 & 0.001143 & 0.49967 & 0.45167 & 0.43185 \\
\hline 0.8 & 0.43997 & 0.001498 & 0.28585 & 0.24529 & 0.22741 \\
\hline 1 & 0.31808 & 0.000575 & 0.16893 & 0.12654 & 0.10915 \\
\hline 2 & -0.37324 & 0.002034 & -0.51960 & -0.56024 & -0.57691 \\
\hline 5 & -2.77600 & 0.000682 & -2.92460 & -2.96510 & -2.98170 \\
\hline 10 & -7.14520 & 0.002161 & -7.29770 & -7.33940 & -7.35590 \\
\hline 20 & -16.31200 & 0.001250 & -16.47400 & -16.51800 & -16.58900 \\
\hline 50 & -44.83400 & 0.001380 & -45.00700 & -45.05200 & \\
\hline 100 & -93.35100 & 0.003623 & -93.53500 & -93.58200 & \\
\hline
\end{tabular}

Therefore, the minimal energy of two noninteracting free electrons that possess no kinetic energy along the magnetic field is $E_{2 e^{-}}(B)=B$, which corresponds to the doubleionization threshold of helium with a fixed nucleus in a magnetic field. In order to get the binding energies $E_{\text {bin }}(B)$ of the helium states, this energy $E_{2 e^{-}}(B)$ has to be subtracted from the total energies:

$$
E_{\mathrm{bin}}(B)=E(B)-E_{2 e^{-}}(B)=E(B)-B .
$$

Figure 1 shows the binding energies for the resonance states belonging to the subspaces $M=0$ (a) and $M=-1$ (b). The absolute values of the binding energies increase with increasing field strength for all resonant states considered here. The rate of increase, however, is larger for the resonances belonging to the symmetry subspace $M=-1$. Additionally, the binding energies of the $\mathrm{He}^{+}$ions $E_{\mathrm{He}^{+}}(B)$ emerging from the field-free quantum number $n=2$ and magnetic quantum numbers $m=0$ (a) and $m=-1$ (b) are shown. The total energies $E_{\mathrm{He}^{+} \text {,tot }}(B)$ of $\mathrm{He}^{+}$in a magnetic field can be obtained via a scaling relation [10] from the precisely known energies of hydrogen in a magnetic field $[10,43]$. In order to get the binding energies $E_{\mathrm{He}^{+}}(B)$ of the $\mathrm{He}^{+}$ion [similar as in Eq. (6)], $E_{e^{-}}(B)$ has to be subtracted from the total energies $E_{\mathrm{He}^{+}, \mathrm{tot}}(B)$ :

$$
E_{\mathrm{He}^{+}}(B)=E_{\mathrm{He}^{+}, \text {tot }}(B)-E_{e^{-}}(B)=E_{\mathrm{He}^{+}, \text {tot }}(B)-B / 2 .
$$

One observes that the binding energies of $\mathrm{He}^{+}\left(2 p_{0}\right)$ and $\mathrm{He}^{+}\left(2 p_{-1}\right)$ correspond to upper thresholds for $E_{\text {bin }}(B)$ of the calculated states with $M=0$ (a) and $M=-1$ (b), respectively [47], and they show the same qualitative behavior as the energies $E_{\mathrm{bin}}(B)$ of our resonances with increasing field strength. This can be interpreted in the following way: as a rough approximation, one of the electrons of the DE helium occupies the corresponding orbital of the $\mathrm{He}^{+}$ion mentioned above. In order to analyze the behavior of the second electron we subtract from the binding energies of the DE helium atoms the corresponding $\mathrm{He}^{+}$energy $E_{\mathrm{He}^{+}}(B)$ :

$$
E_{2}(M, B)=E_{\text {bin }}(M, B)-E_{\mathrm{He}^{+}}\left(2 p_{M}, B\right) .
$$

The corresponding results are shown in Fig. 2. One observes a qualitatively different behavior of the energy levels according to three different regimes: (I) low-field regime $0 \leqslant B \leqslant 10^{-2}$, (II) intermediate-field regime $10^{-2}<B \leqslant 1$, and (III) high-field regime $1<B \leqslant 10^{2}$. Especially in (I) but also in (III) there is a comparatively weak dependence on the magnetic field. On the other hand, in (II) a reorganization of the energy levels takes place being most pronounced in Fig. 2(a). This behavior is also known for hydrogen and singly excited helium in a magnetic field. In both subfigures three 
TABLE VIII. Calculated energies $E$ and resonance widths $\Gamma$ of resonances of the subspace ${ }^{3}(-1)^{+}$and $N=2$ for different magnetic field strengths $B: 1^{3}(-1)^{+} 2^{3}(-1)^{+}, 3^{3}(-1)^{+}$, and $4^{3}(-1)^{+}$[field-free ${ }^{3} P_{2}^{o}(1,0)_{2}^{+},{ }^{3} P_{2}^{o}(1,0)_{3}^{+},{ }^{3} P^{o}{ }_{2}(0,1)_{3}^{-}$, and $\left.{ }^{3} F_{2}^{o}{ }_{2}(1,0)_{3}^{0}\right]$.

\begin{tabular}{|c|c|c|c|c|c|}
\hline \multirow[b]{2}{*}{$B$} & \multicolumn{2}{|c|}{$1^{3}(-1)^{+}$} & \multirow{2}{*}{$\begin{array}{c}2^{3}(-1)^{+} \\
-E\end{array}$} & \multirow{2}{*}{$\begin{array}{c}3^{3}(-1)^{+} \\
-E\end{array}$} & \multirow{2}{*}{$\begin{array}{c}4^{3}(-1)^{+} \\
-E\end{array}$} \\
\hline & $E$ & $\Gamma / 2$ & & & \\
\hline 0 & $0.76049[36]$ & 0.000149 & $0.58465[36]$ & $0.57900[36]$ & $0.56568[41]$ \\
\hline 0 & 0.76044 & 0.000150 & 0.58465 & 0.57900 & 0.56568 \\
\hline 0.0002 & 0.76055 & 0.000123 & 0.58475 & 0.57908 & 0.56608 \\
\hline 0.0005 & 0.76059 & 0.000090 & 0.58484 & 0.57913 & 0.56571 \\
\hline 0.001 & 0.76092 & 0.000224 & 0.58498 & 0.57905 & 0.56541 \\
\hline 0.002 & 0.76121 & 0.000117 & 0.58542 & 0.57985 & 0.56701 \\
\hline 0.005 & 0.76284 & 0.000163 & 0.58682 & 0.58115 & 0.56777 \\
\hline 0.01 & 0.76516 & 0.000140 & 0.58861 & 0.58313 & 0.56994 \\
\hline 0.02 & 0.76911 & 0.000611 & 0.59056 & 0.58572 & 0.57277 \\
\hline 0.04 & 0.77664 & 0.000123 & 0.59090 & 0.58756 & 0.57433 \\
\hline 0.08 & 0.78635 & 0.000123 & 0.59067 & 0.58004 & 0.57017 \\
\hline 0.1 & 0.78735 & 0.000161 & 0.58934 & 0.56246 & 0.54601 \\
\hline 0.16 & 0.78813 & 0.000172 & 0.58511 & 0.55843 & 0.53253 \\
\hline 0.4 & 0.70867 & 0.000172 & 0.51165 & 0.45580 & 0.44592 \\
\hline 0.8 & 0.47094 & 0.000102 & 0.29665 & 0.24898 & 0.22976 \\
\hline 1 & 0.33918 & & 0.17395 & 0.12839 & 0.11003 \\
\hline 2 & -0.36680 & 0.000106 & -0.51867 & -0.55994 & -0.57678 \\
\hline 5 & -2.77240 & & -2.92410 & -2.96500 & -2.98160 \\
\hline 10 & -7.14050 & 0.000067 & -7.29700 & -7.33870 & -7.35560 \\
\hline 20 & -16.3110 & & -16.47500 & -16.51800 & -16.59300 \\
\hline 50 & -44.8330 & & -45.0080 & -45.0520 & -45.070000 \\
\hline 100 & -93.3510 & 0.000302 & -93.5350 & -93.58200 & \\
\hline
\end{tabular}

additional energies are shown (dashed line). These approximate energies $E_{\mathrm{app}(B)}$ are the sum of the binding energy $E_{\mathrm{He}^{+}\left(2 p m_{\mathrm{He}^{+}}\right)}(B)$ of the helium ion $\mathrm{He}^{+}\left(2 p_{0}\right)$ (a) and $\mathrm{He}^{+}\left(2 p_{-1}\right)$ (b) and the energetically lowest binding energies $E_{\mathrm{H}\left(n m_{\mathrm{H}}\right)}(B)$ of the hydrogen atom with $n \geqslant 2$ :

$$
E_{\text {app }}(B)=E_{\mathrm{He}^{+}\left(2 p m_{\mathrm{He}^{+}}\right)}(B)+E_{\mathrm{H}\left(n l m_{\mathrm{H}}\right)}(B) .
$$

The combination of the individual quantum numbers is in agreement with the total quantum numbers of the considered subspace:

$$
M=m_{\mathrm{He}^{+}}+m_{\mathrm{H}} \quad \text { and } \quad \Pi_{z}=\left(\pi_{\mathrm{He}^{+}}\right)_{z}\left(\pi_{\mathrm{H}}\right)_{z} .
$$

In these approximate energies, no interaction between the two electrons is taken into account, and a complete screening of one charge unit of the nucleus by the inner electron is assumed. Considering the subspace $M=-1$ in Fig. 2(b) one can see that in the high-field regime (III) the energies $E_{\text {app }}(B)$ come energetically close to the calculated resonance energies and show the same qualitative behavior. In this regime also a degeneracy of the singlet (solid line) and triplet (dotted line) states takes place (only $S_{z}=0$ is considered here). These features become more pronounced with increasing field strength and higher degree of excitation (logarithmic scale). Let us concentrate as an example on the approximation $E_{\text {app }}\left(\mathrm{He}^{+}\left(2 p_{-1}\right) \mathrm{H}\left(2 s_{0}\right)\right)$ to the energies of the singlet and triplet ground states $1^{1}(-1)^{+}$and $1^{3}(-1)^{+}$, respectively. It obviously significantly underestimates the magnitude of their binding energies in the field regimes (I) and (II) where both states are also energetically significantly separated. This is a sign of the strong overlap of the two electrons of the DE helium. This overlap decreases in the region (III), and the electrons become spatially separated. Therefore, it is possible in (III) to speak of an "inner" $\mathrm{He}^{+}$-like electron, which is located close to the nucleus and screens one charge unit, and of an "outer" H-like electron at a distance noticing only one effective positive charge.

The above-observed behavior can be illustrated by using an independent-particle picture. All one-electron states except the tightly bound states are elongated along the magnetic field axis and squeezed perpendicular to it with increasing magnetic field strength. Tightly bound states $\left(1 s_{0}, 2 p_{-1}, 3 d_{-2}, \ldots\right)$ are confined parallel to the magnetic field axis as well, and their binding energy rises strongly with increasing field strength [10]. In the case that one of the electrons occupies a tightly bound orbital, the overlap of two electrons steadily decreases with increasing magnetic field strength. Thus the tightly bound electron is located close to the nucleus screening one charge, while the second electron 
TABLE IX. Calculated energies $E$ and resonance widths $\Gamma$ of resonances of the subspace ${ }^{1}(-2)^{+}$and $N=2$ for different magnetic field strengths $B$ : $1^{1}(-2)^{+}$and $2^{1}(-2)^{+}$[field-free ${ }^{1} D^{e}{ }_{2}(1,0)_{2}^{+}$and $\left.{ }^{1} D^{e}{ }_{2}(1,0)_{3}^{+}\right]$.

\begin{tabular}{lcccc}
\hline \hline & \multicolumn{2}{c}{$1^{1}(-2)^{+}$} & \multicolumn{2}{c}{$2^{1}(-2)^{+}$} \\
\cline { 2 - 5 }$B$ & $-E$ & $\Gamma / 2$ & $-E$ & $\Gamma / 2$ \\
\hline 0 & $0.70195[37]$ & 0.001183 & $0.56922[37]$ & 0.000278 \\
\hline 0 & 0.70157 & 0.001121 & 0.56913 & 0.000261 \\
0.0005 & 0.70203 & 0.001122 & 0.56960 & 0.000261 \\
0.001 & 0.70249 & 0.001125 & 0.57009 & 0.000264 \\
0.002 & 0.70249 & 0.001125 & 0.57009 & 0.000264 \\
0.005 & 0.70643 & 0.001052 & 0.57379 & 0.000249 \\
0.01 & 0.71116 & 0.001105 & 0.57755 & 0.000270 \\
0.02 & 0.72055 & 0.001152 & 0.58413 & 0.000283 \\
0.05 & 0.74541 & 0.001196 & 0.59369 & 0.000308 \\
0.1 & 0.77727 & 0.001020 & 0.59346 & 0.000433 \\
0.16 & 0.80518 & 0.001557 & 0.58362 & 0.000615 \\
0.5 & 0.82534 & 0.004077 & 0.47937 & 0.000573 \\
1 & 0.68014 & 0.006971 & 0.21906 & 0.000840 \\
2 & 0.16923 & 0.005071 & -0.44999 & 0.000117 \\
5 & -1.92293 & 0.007648 & -2.83753 & 0.001424 \\
10 & -5.97779 & 0.002951 & -7.19591 & 0.000081 \\
20 & -14.78130 & & -16.35960 & 0.000128 \\
50 & -42.51036 & & -44.87839 & \\
100 & -90.36262 & & -93.39070 & 0.000145 \\
\hline \hline & & & &
\end{tabular}

stays mainly near the $z$ axis at a distance to the core and behaves therefore similar to an electron of a hydrogen atom. This scenario applies to the subspace $M=-1 \Pi_{z}=+1$ since $2 p_{-1}$ is a tightly bound orbital and consequently all other possible orbitals of the second electron are not tightly bound.

In the subspace $M=0$ in Fig. 2(a) no degeneracy of the energies of the singlet and triplet states occurs in the presence of a strong field. However, the singlet states can be well approximated by the corresponding $E_{\text {app }}(B)$. An exception to this rule is the ground state $1^{1}(0)^{+}$, which is approximated only qualitatively by the binding energies of $\operatorname{He}^{+}\left(2 p_{0}\right)$ and $\mathrm{H}\left(2 p_{0}\right)$, which corresponds to an intrashell state. In an intrashell state with both electrons approximately in the same orbital, it is expected that the assumption of weakly interacting electrons fails. Of course, there is no triplet partner for the ground state which is approximately described by the symmetric $\left(2 p_{0}\right)^{2}$ configuration. In contrast to the subspace $M=-1$ the resonances of the subspace $M=0$ contain no (dominating) configurations that include a tightly bound orbital. As a consequence also the "inner" $\mathrm{He}^{+}$-like electron is elongated along the $z$ axis and a noticeable overlap of the electronic orbitals remains even in the strong-field regime (III).

The strong increase of some energies in the regime $0.1 \leqslant B \leqslant 1$ [see Figs. 2(a) and 2(b)] can be partly modeled with two-electron configurations that consist of an electronic wave function of a helium ion $\operatorname{He}^{+}\left(2 s_{0}\right)$ and a hydrogen atom and are not shown in Fig. 2. This effect results from the
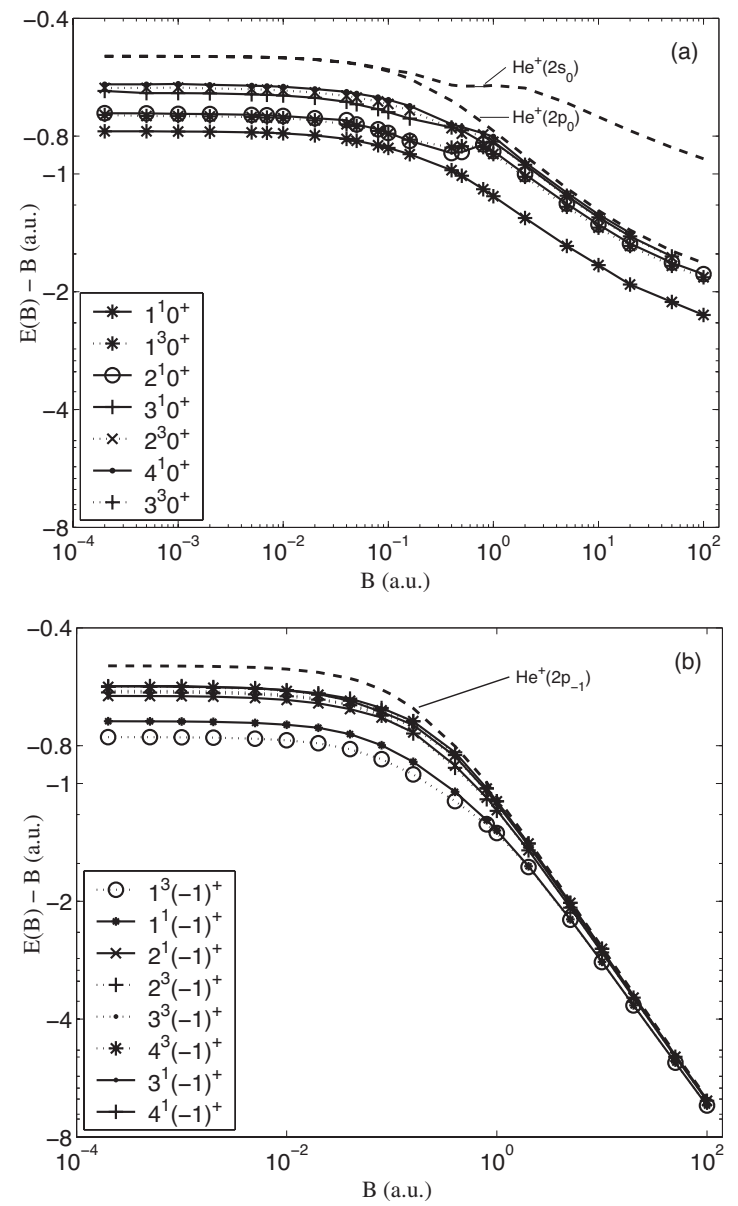

FIG. 1. Binding energies $E_{\text {bin }}(B)=E(B)-B$ of doubly excited helium in a magnetic field range $0.0002 \leqslant B \leqslant 100$. The dependence on the magnetic field is shown for the energetically lowest resonances of the subspaces $M=0, \Pi_{z}=+1$ (a) and $M=-1, \Pi_{z}=+1$ (b). Solid lines stand for singlet and dotted lines for triplet states. Additionally, appropriate binding energies of $\mathrm{He}^{+}$ions are given (dashed lines) which correspond to upper thresholds.

increasing energetic gap between the binding energy of $\mathrm{He}^{+}\left(2 s_{0}\right)$ and $\mathrm{He}^{+}\left(2 p_{0}\right)$ which can be seen in Fig. 1(a). Note that the approximations applied in the strong-magnetic-field regime (III) do not hold in the regimes (I) and (II) and particularly not in the field-free situation [40].

As a next step we aim at investigating the energetically lowest DE helium state in a strong magnetic field. Therefore, we searched for the DE helium state with the strongest binding energy in the field range (III). The latter is dominated by the configuration $\mathrm{He}^{+}\left(2 p_{-1}\right) \mathrm{H}\left(2 p_{-1}\right)$ [10]. Consequently we calculated the energetically lowest singlet states of the subspace $M=-2$ and $\Pi_{z}=+1$.

In Fig. 3 the energetic distances of the two energetically lowest singlet states to the first ionization threshold $I_{1}(B)$ $=E_{\mathrm{He}^{+}\left(1 s_{0}\right)}(B)+B$ are shown. The energies of both states show a weak dependence on the field strength for $B<0.01$. For $B>0.01$ the energetic difference of the energetically lowest resonance state $1^{1}(-2)^{+}$to $I_{1}(B)$ decreases with increasing field strength. A crossover of the first ionization threshold seems to take place for $B \approx 65$ a.u. This would correspond to 

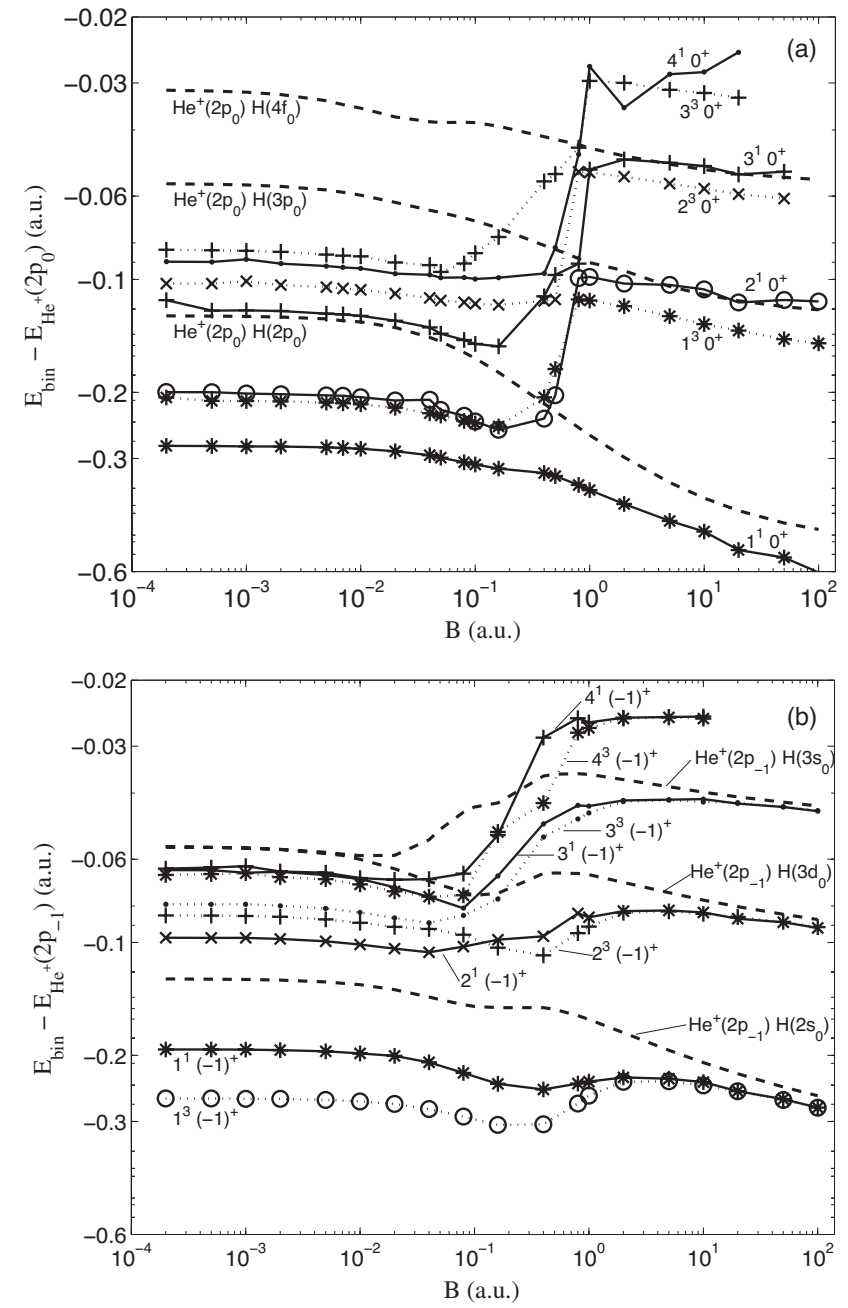

FIG. 2. Differences of the binding energies of the doubly excited helium atom and the $\mathrm{He}^{+}$ion $E_{\text {bin }}(B)-E_{\mathrm{He}^{+}}(B)$ in a magnetic field range $0.0002 \leqslant B \leqslant 100$. The dependence on the magnetic field is shown for the energetically lowest states of the subspaces $M=0$, $\Pi_{z}=+1$ (a) and $M=-1, \Pi_{z}=+1$ (b). Solid lines stand for singlet and dotted lines for triplet states. Additionally to $E_{\mathrm{bin}}(B)$, approximate energies are given (dashed lines) which are a sum of the binding energies of $\mathrm{a} \mathrm{He}^{+}$ion and a hydrogen atom (see text for details).

a stabilization of the resonance $1^{1}(-2)^{+}$and a conversion to a bound state for a magnetic field $B>65$ since the autoionization process is not possible anymore. The energetically lowest resonance is approximated only qualitatively by the configuration involving the noninteracting orbitals $\operatorname{He}^{+}\left(2 p_{-1}\right)$ and $\mathrm{H}\left(2 p_{-1}\right)$, for it is an intrashell state which consists of two tightly bound orbitals. We note that the above considerations assume a spin singlet for both the resonance and threshold states, which is indeed not the energetically lowest state in the strong-field regime.

The second singlet state $2^{1}(-2)^{+}$is approximated well by the configuration involving the orbitals $\mathrm{He}^{+}\left(2 p_{-1}\right)$ and $\mathrm{H}\left(3 p_{-1}\right)$, and for $B>1$ there is hardly a difference visible on

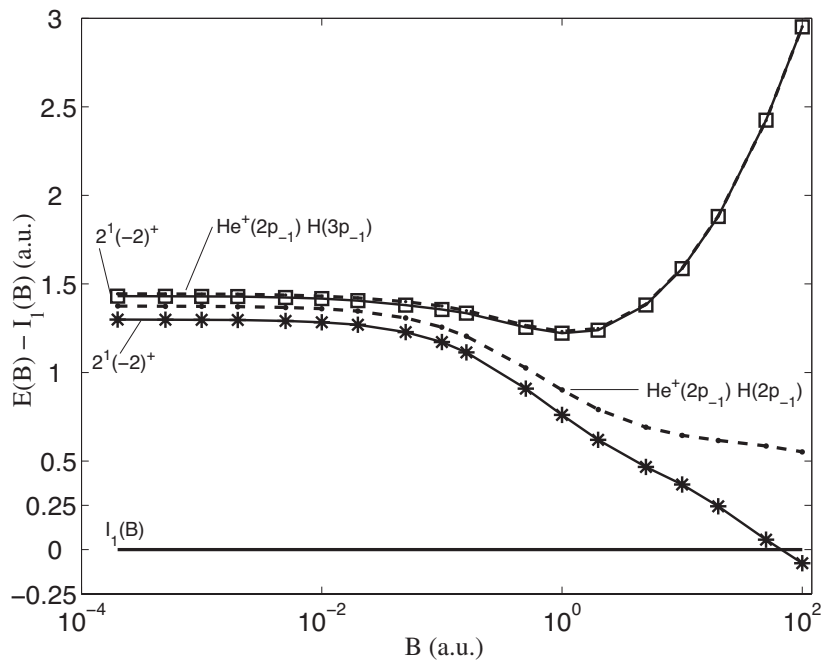

FIG. 3. Energetic difference $E(B)-I_{1}(B)$ of DE helium energies to the first ionization threshold $I_{1}(B)$. The energies of the two energetically lowest singlet states of the subspace $M=-2, \Pi_{z}=+1$ in a magnetic field range $0.0002 \leqslant B \leqslant 100$ are presented. Additionally, approximate energies (dashed lines) are given which are a sum of the binding energies of a $\mathrm{He}^{+}$ion and a hydrogen atom.

the scale of Fig. 3. This can be explained by applying the same argument as for the subspace $M=-1$ where all calculated states also consist approximately of a tightly bound and a nontightly bound orbital. However, the energetic difference to $I_{1}(B)$ increases strongly in the high-field regime and no crossing of $I_{1}(B)$ is possible.

Using the results which have been obtained so far one can relate the doubly excited helium states in a magnetic field denoted by the spectroscopic field notation (2) in the limit of a high-field strength to the corresponding dominant independent-particle configurations of a helium ion and hydrogen electron. This has been done in Table III. In addition, the corresponding correlation quantum numbers (4) for a vanishing magnetic field $B=0$ are given.

\section{Resonance widths}

Parallel to the real energies dealt with in Sec. IV B 2, the corresponding imaginary parts $\Gamma$ have been calculated, too. $\Gamma$ is interpreted as the width of the resonance and therefore as the inverse lifetime. Typically, the absolute values of the widths are by orders of magnitude smaller than the real energies. Our method allows for the same absolute accuracy for both the real and imaginary parts. This leads to a significantly lower relative accuracy of the resonance widths compared to the real energies. The widths themselves vary by orders of magnitudes. Therefore, it is most natural to inspect the behavior of those widths more closely which possess a large absolute value $(\Gamma / 2 \geqslant 0.0001)$. These include three singlet states of the $M=0, \Pi_{z}=+1$ subspace $1^{1} 0^{+}, 2^{1} 0^{+}$, and $4^{1} 0^{+}$, which are shown in Fig. 4(a). Additionally, the widths of the two energetically lowest resonance states of the 

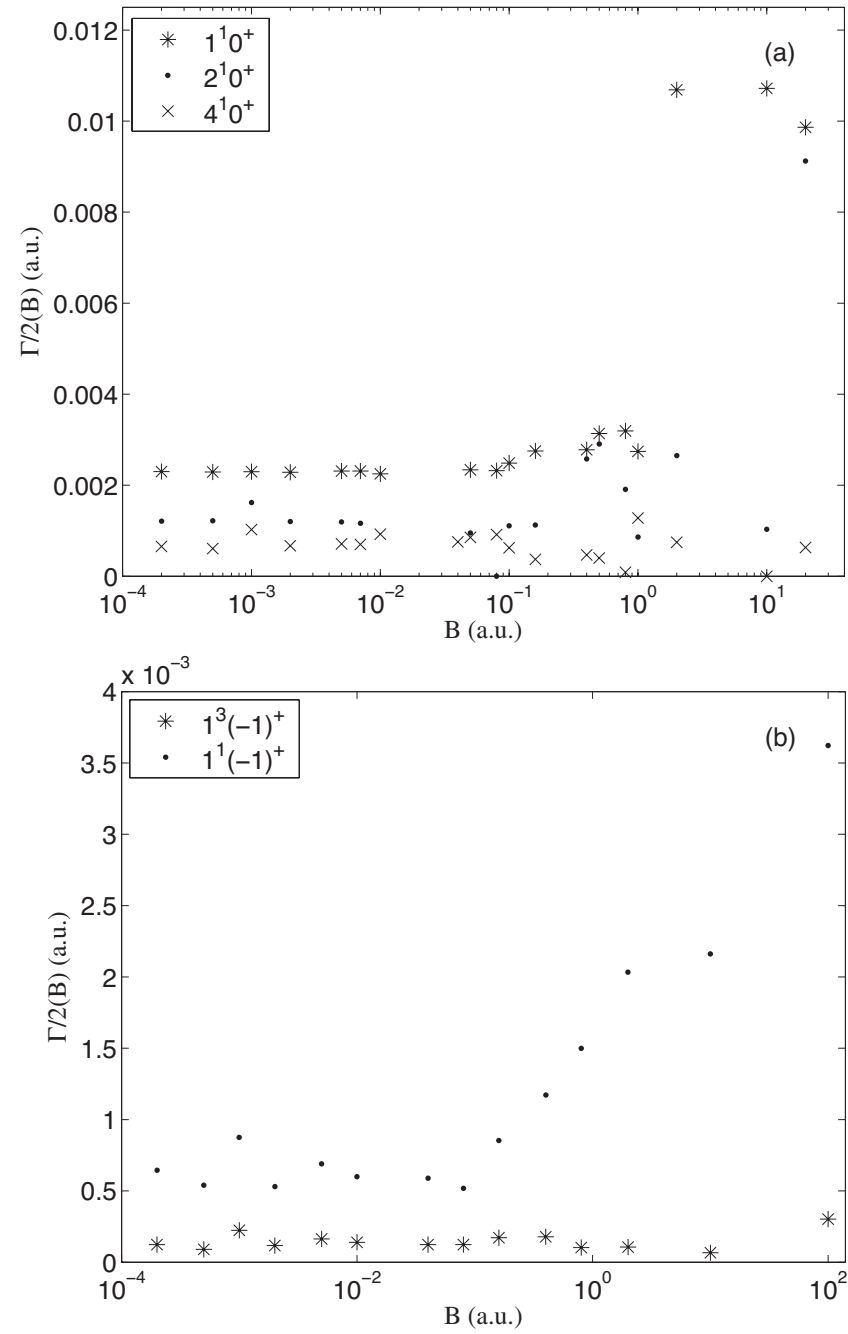

FIG. 4. Resonance widths $\Gamma$ of the DE helium atom in a magnetic field range $0.0002 \leqslant B \leqslant 100$. The widths of three singlet states with $M=0, \Pi_{z}=+1$ (a) and of the two ground states with $M= \pm 1$, $\Pi_{z}=+1$ (b) are plotted.

$M=-1, \Pi_{z}=+1$ subspace, $1^{1}(-2)^{+}$and $1^{3}(-2)^{+}$, are presented in Fig. 4(b). The corresponding data can be found in Tables IV, V, and VII-IX.

For $B<0.05$ just a weak dependence on the magnetic field can be observed for the three singlet states in Fig. 4(a). With further increasing field strength, the widths start to fluctuate, although this fluctuation could possibly be a consequence of the finite numerical accuracy. The width of the state $1^{1} 0^{+}$increases in the regime $1<B<2$ approximately by a factor of 4 .

In Fig. 4(b) we compare a singlet and a triplet state otherwise possessing the same quantum numbers. In Fig. 2(b) we see that the energies of these states become degenerate for $B>2$. For $B<0.1$ their widths fluctuate around a mean value of $7 \times 10^{-4}\left[1^{1}(-1)^{+}\right]$and $1.5 \times 10^{-4}\left[1^{3}(-1)^{+}\right]$, respectively. For $B>0.1$ the width of the triplet state keeps fluctuating, but the width of the singlet states increases with magnetic field strength $B$. This means that both states become energetically degenerate for strong magnetic fields, but the behavior of the corresponding resonance widths is different in this field range.

\section{SUMMARY AND OUTLOOK}

We have investigated the energies and lifetimes of the resonance states of a doubly excited helium atom exposed to a magnetic field. The nonrelativistic, infinite-mass Hamiltonian is characterized by the symmetries associated with the four quantum numbers: $M, \Pi_{z}, S^{2}$, and $S_{z}$. Our computational approach consists of the complex scaling method applied to a full configuration-interaction scheme based on nonlinearly optimized anisotropic Gaussian orbitals.

In order to show the behavior of the DE helium states denoted by the spectroscopic field notation (2) in the limits of weak and strong magnetic field strengths, we have presented for vanishing magnetic field their corresponding correlation quantum numbers (4) and for a strong magnetic field their corresponding independent-particle configurations of a helium ion and hydrogen electron.

In total the energies of $17 \mathrm{DE}$ states have been calculated. Their behavior can be subdivided into three magnetic field ranges: the weak- $\left(0 \leqslant B \leqslant 10^{-2}\right)$, the intermediate- $\left(10^{-2}\right.$ $<B \leqslant 1)$, and the strong- $\left(1<B \leqslant 10^{2}\right)$ field regimes. With increasing field strength we observe the transition from a weak to a strong dependence on magnetic field which is accompanied by a reorganization of the energies of the resonances especially in the intermediate-field regime.

In the strong-field regime, a clustering of the calculated energies into different branches according to their magnetic quantum numbers takes place. Furthermore, the electronelectron interaction becomes less important with increasing field strength, being more pronounced for the states of subspace $M=-1$ than for those of $M=0$. As a result, a crude description of the resonance energies by the sum of the energies of a $\mathrm{He}^{+}$ion and a hydrogen atom is possible. An exception to this rule is the intrashell states. The DE helium state with the lowest energy $\left(S_{z}=0\right)$ is the state $1^{1}(-2)^{+}$of the subspace $M=-2$. This resonance state can probably be stabilized by a magnetic field, for its calculated energy becomes smaller than the one-electron ionization threshold for a field strength of $B>65$.

The behavior of the widths of five calculated resonances with large absolute value $\left(\Gamma \geqslant 10^{-4}\right)$ has been inspected. The dependence of the widths on the magnetic field for low field strengths $B<0.1$ is weak. The values of the widths are fluctuating around their mean values, which could be due to the finite numerical accuracy of our method. For field strengths $B>1$ the widths of two states-namely, $1^{1} 0^{+}$and $1^{1}(-1)^{+}$- significantly increase while the widths of the other investigated states still fluctuate with an increasing amplitude.

Having presented the first data on the energies of the resonances of DE helium in a magnetic field, several issues still have to be addressed in future works. In order to get synthetic spectra of astronomical objects like MWD's, the number of calculated states should be increased including also 
states with $\Pi_{z}=-1$. Additionally, magnetic-field-dependent oscillator strengths and electromagnetic-transition probabilities have to be studied. On the other hand, a closer look at the resonance widths would be desirable in order to gain a deeper understanding of the role of electron-electron interactions and the autoionization process under the influence of a magnetic field. An increased accuracy of our data would be a prerequisite for this.

\section{ACKNOWLEDGMENT}

We are grateful to H.-D. Meyer for enlightening discussions.
[1] D. Reimers, S. Jordan, V. Beckmann, N. Christlieb, and L. Wisotzki, Astron. Astrophys. 337, L13 (1998).

[2] G. D. Schmidt et al., Astrophys. J. 595, 1101 (2003).

[3] K. M. Vanlandingham et al., Astron. J. 130, 734 (2005).

[4] F. Euchner et al., Astron. Astrophys. 451, 671 (2006).

[5] J. Trümper et al., Astrophys. J. 219, L105 (1978).

[6] H. Friedrich and D. Wintgen, Phys. Rep. 183, 37 (1989).

[7] L. Schiff and H. Snyder, Phys. Rev. 55, 59 (1939).

[8] R. Henry and R. O'Connell, Astrophys. J. 282, L97 (1984).

[9] H. Forster, W. Strupat, W. Rösner, G. Wunner, H. Ruder, and H. Herold, J. Phys. B 17, 1301 (1984).

[10] H. Ruder, G. Wunner, H. Herold, and F. Geyer, Atoms in Strong Magnetic Fields (Springer, Heidelberg, 1994).

[11] J. P. R. Angle, J. Liebert, and H. S. Liebert, Astrophys. J. 292, 260 (1985).

[12] J. L. Greenstein, R. J. W. Henry, and R. F. O'Connell, Astrophys. J. 289, L25 (1985).

[13] D. T. Wickramasinghe and L. Ferrario, Publ. Astron. Soc. Pac. 112, 873 (2000).

[14] W. Becken, P. Schmelcher, and F. K. Diakonos, J. Phys. B 32, 1557 (1999).

[15] W. Becken and P. Schmelcher, J. Phys. B 33, 545 (2000).

[16] W. Becken and P. Schmelcher, Phys. Rev. A 63, 53412 (2001).

[17] S. Jordan, P. Schmelcher, and W. Becken, Astron. Astrophys. 376, 614 (2001).

[18] O.-A. Al-Hujaj and P. Schmelcher, Phys. Rev. A 67, 23403 (2003).

[19] S. Jordan, P. Schmelcher, W. Becken, and W. Schweizer, Astron. Astrophys. 336, L33 (1998).

[20] N. Merani, J. Main, and G. Wunner, Astron. Astrophys. 298, 193 (1995).

[21] O.-A. Al-Hujaj and P. Schmelcher, Phys. Rev. A 70, 33411 (2003).

[22] O.-A. Al-Hujaj and P. Schmelcher, Phys. Rev. A 70, 23411 (2003).

[23] B. R. Johnson, J. O. Hirschfelder, and K. H. Yang, Rev. Mod. Phys. 55, 109 (1983).

[24] P. Schmelcher, L. S. Cederbaum, and U. Kappes, Conceptual Trends in Quantum Chemistry (Kluwer, Dordrecht, 1994), pp. $1-51$.

[25] P. Schmelcher and L. S. Cederbaum, Phys. Rev. A 43, 287 (1991).

[26] P. Schmelcher and L. S. Cederbaum, Phys. Rev. A 37, 672 (1988).

[27] U. Kappes and P. Schmelcher, Phys. Rev. A 51, 4542 (1995); 54, 1313 (1996); Phys. Lett. A 210, 409 (1996).

[28] T. Detmer, P. Schmelcher, F. K. Diakonos, and L. S. Cederbaum, Phys. Rev. A 56, 1825 (1997); T. Detmer, P. Schmelcher, and L. S. Cederbaum, ibid. 57, 1767 (1998).

[29] O.-A. Al-Hujaj and P. Schmelcher, Phys. Rev. A 61, 63413 (2000).

[30] O.-A. Al-Hujaj and P. Schmelcher, Phys. Rev. A 68, 53403 (2003).

[31] W. P. Reinhardt, Annu. Rev. Phys. Chem. 33, 223 (1982).

[32] N. Moiseyev, Phys. Rep. 302, 211 (1998).

[33] N. Moiseyev, P. R. Certain, and F. Weinhold, Mol. Phys. 36, 1613 (1978).

[34] G. Tanner, K. Richter, and J.-M. Rost, Rev. Mod. Phys. 72, 497 (2000).

[35] A. Bürgers, D. Wintgen, and J.-M. Rost, J. Phys. B 28, 3163 (1995)

[36] Y. K. Ho, Phys. Rev. A 48, 3598 (1993).

[37] Y. K. Ho and A. K. Bhatia, Phys. Rev. A 44, 2895 (1991).

[38] H. Bachau, At. Data Nucl. Data Tables 48 t167 (1991).

[39] D. R. Herrick and O. Sinanoğlu, Phys. Rev. A 11, 97 (1975).

[40] C. D. Lin, Phys. Rev. A 29, 1019 (1983).

[41] E. Lindroth, Phys. Rev. A 49, 4473 (1994).

[42] L. D. Landau and E. M. Lifshitz, Quantum Mechanics: NonRelativistic Theory (Butterworth-Heinemann, Oxford, 1997).

[43] Y. P. Kravchenko, M. A. Liberman, and B. Johansson, Phys. Rev. A 54, 287 (1996).

[44] European Southern Observatory.

[45] Sloan Digital Sky Survey.

[46] http://www.astronomy.villanova.edu/WDCatalog/index.html

[47] The quantum number $n$ and the angular momentum $l$ are not good quantum numbers for $B>0$. However, they could be used for finite field strength as labels for states having these quantum numbers for $B=0$ [10]. 